\title{
Quantum Graphs as Quantum Relations
}

\author{
Nik Weaver ${ }^{1}$ \\ Accepted: 20 April 2019 / Published online: 13 January 2021 \\ (c) The Author(s) 2021
}

\begin{abstract}
The "noncommutative graphs" which arise in quantum error correction are a special case of the quantum relations introduced in Weaver (Quantum relations. Mem Am Math Soc 215(v-vi):81-140, 2012). We use this perspective to interpret the KnillLaflamme error-correction conditions (Knill and Laflamme in Theory of quantum error-correcting codes. Phys Rev A 55:900-911, 1997) in terms of graph-theoretic independence, to give intrinsic characterizations of Stahlke's noncommutative graph homomorphisms (Stahlke in Quantum zero-error source-channel coding and noncommutative graph theory. IEEE Trans Inf Theory 62:554-577, 2016) and Duan, Severini, and Winter's noncommutative bipartite graphs (Duan et al., op. cit. in Zeroerror communication via quantum channels, noncommutative graphs, and a quantum Lovász number. IEEE Trans Inf Theory 59:1164-1174, 2013), and to realize the noncommutative confusability graph associated to a quantum channel (Duan et al., op. cit. in Zero-error communication via quantum channels, noncommutative graphs, and a quantum Lovász number. IEEE Trans Inf Theory 59:1164-1174, 2013) as the pullback of a diagonal relation. Our framework includes as special cases not only purely classical and purely quantum information theory, but also the "mixed" setting which arises in quantum systems obeying superselection rules. Thus we are able to define noncommutative confusability graphs, give error correction conditions, and so on, for such systems. This could have practical value, as superselection constraints on information encoding can be physically realistic.
\end{abstract}

Keywords Quantum graphs · Quantum relations · Quantum error correction

Mathematics Subject Classification Primary 47L25 - 81P45 - 81P47; Secondary 05C $90 \cdot 46 \mathrm{~L} 10$

\footnotetext{
Partially supported by NSF Grant DMS-1067726.

$凶$ Nik Weaver

nweaver@math.wustl.edu

1 Department of Mathematics, Washington University, Saint Louis, MO 63130, USA 


\section{Quantum Graphs and Quantum Relations}

"Quantum" or "noncommutative" graphs appear in the setting of quantum error correction $[3,10,11]$. The usual construction starts with a quantum channel, which in the Schrodinger picture is modelled by a completely positive trace preserving (CPTP) map $\Phi: M_{m} \rightarrow M_{n}$. Here $M_{m}$ is the set of $m \times m$ complex matrices and a CPTP map is concretely realized as a linear map of the form

$$
\Phi: \rho \rightarrow \sum_{i} K_{i} \rho K_{i}^{*}
$$

where $\rho \in M_{m}$ and the Kraus matrices $K_{i}$ are a finite family of $n \times m$ matrices satisfying $\sum K_{i}^{*} K_{i}=I_{m}$ (the $m \times m$ identity matrix).

Positive matrices with unit trace represent mixed states, and pure states appear as the special case of matrices of the form $|\alpha\rangle\langle\alpha|$ for $|\alpha\rangle$ a unit vector in $\mathbb{C}^{m}$. Thus quantum channels transform mixed states to mixed states, and in error correction problems one is interested in determining which input states can be distinguished with certainty after passing through the channel. The condition that the images of two pure states $|\alpha\rangle$ and $|\beta\rangle$ after transmission must be orthogonal can be expressed as

$$
\langle\alpha|B| \beta\rangle=0 \quad \text { for all } B \in \mathcal{V}_{\Phi}=\operatorname{span}\left\{K_{i}^{*} K_{j}\right\}
$$

(see, e.g., [3]).

The space $\mathcal{V}_{\Phi} \subseteq M_{m}$ is an operator system-a linear subspace of $M_{m}$ which is stable under the adjoint operation and contains the identity matrix (since we have assumed that $\sum K_{i}^{*} K_{i}=I_{m}$ ). In the analogous classical setting one would be dealing with a finite set of possible input states and one could create a graph by placing an edge between any pair of input states which might, after transmission through a noisy channel, be received as the same output state. This classical confusability graph is relevant to classical zero-error communication in something like the way that the operator system $\mathcal{V}_{\Phi}$ is relevant to zero-error communication in the quantum setting. This led Duan, Severini, and Winter to term $\mathcal{V}_{\Phi}$ a noncommutative confusability graph [3].

Going further, since any operator system can arise in the above manner from a quantum channel, they suggested that operator systems generally could be considered "noncommutative graphs". This daring proposal was supported by the fact that they were able to define a "quantum Lovász $\vartheta$ function" for any operator system, in analogy to the classical Lovász $\vartheta$ function of a graph.

At around the same time, the notion of a "quantum relation" was introduced in [12]. This notion gives rise to natural definitions of such things as "quantum equivalence relations" and "quantum partial orders", and it is also the basis of the "quantum metrics" and "quantum uniform structures" which were studied in [7], an earlier project from which the notion of a quantum relation was extracted. 
The identification of operator systems as "quantum graphs" was also made in [12], but not pursued further there. ${ }^{1}$ However, it is worth investigating this connection, as under the quantum relations point of view basic aspects of the theory of zero-error quantum communication become conceptually transparent.

The core idea is that an operator space - a linear subspace of $M_{m}$ - can be thought of as a quantum analog of a relation on a finite set. (In infinite dimensions, this becomes a weak* closed operator space, but I will stick to the finite-dimensional setting in this paper.) Classically, a relation on a set $X$ is a subset $R$ of $X \times X$, and we write $x R y$ to indicate that the pair $(x, y)$ belongs to the relation. The relation $R$ is said to be

- reflexive if $x R x$, for all $x \in X$

- symmetric if $x R y$ implies $y R x$, for all $x, y \in X$

- antisymmetric if $x R y$ and $y R x$ imply $x=y$, for all $x, y \in X$

- transitive if $x R y$ and $y R z$ imply $x R z$, for all $x, y, z \in X$.

This can be expressed more algebraically by letting $\Delta$ be the diagonal relation $\Delta=$ $\{(x, x): x \in X\}$, letting $R^{t}$ be the transpose relation $R^{t}=\{(y, x):(x, y) \in R\}$, and letting $R R^{\prime}$ be the product relation $R R^{\prime}=\left\{(x, z):(x, y) \in R\right.$ and $(y, z) \in R^{\prime}$ for some $y \in X$ \}. We can then say that $R$ is

- reflexive if $\Delta \subseteq R$

- symmetric if $R=R^{t}$

- antisymmetric if $R \cap R^{t} \subseteq \Delta$

- transitive if $R^{2} \subseteq R$.

The analogous definitions for an operator space $\mathcal{V} \subseteq M_{m}$ characterize $\mathcal{V}$ as

- reflexive if $I_{m} \in \mathcal{V}$

- symmetric if $\mathcal{V}=\mathcal{V}^{*}$

- antisymmetric if $\mathcal{V} \cap \mathcal{V}^{*} \subseteq \mathbb{C} \cdot I_{m}$

- transitive if $\mathcal{V}^{2} \subseteq \mathcal{V}$.

We define $\mathbb{C} \cdot I_{m}$ to be the diagonal quantum relation on $M_{m}$, so that $\mathcal{V}$ is reflexive if and only if $\mathbb{C} \cdot I_{m} \subseteq \mathcal{V}$, in closer analogy with the classical case. In the above, $\mathcal{V}^{*}=\left\{A^{*}: A \in \mathcal{V}\right\}$ is the set of Hermitian adjoints of matrices in $\mathcal{V}$ and $\mathcal{V}^{2}=$ $\operatorname{span}\{A B: A, B \in \mathcal{V}\}$ is a special case of the product of two operator spaces.

Graphs appear in this framework by regarding a classical graph as a set of vertices equipped with a reflexive, symmetric relation. The elements of the relation represent edges, and symmetry expresses the fact that edges are undirected. Reflexivity corresponds to the convention that there is a loop at each vertex. This makes sense in the error correction setting: if we place an edge between any two states which might be confused, then it is natural to include an edge between any state and itself. (More pointedly, it is unnatural, and creates unnecessary complication, not to do this.) Of course, in other settings we may not wish to impose this requirement, in which case we could drop reflexivity and define a quantum graph to merely be a symmetric quantum relation. This was the approach taken in [11]. For the sake of definiteness, I will use the

\footnotetext{
1 The expression "quantum graph" unhappily conflicts with an earlier, unrelated use of this term, and also with the "noncommutative graph" terminology used in [3]. But in a setting that also includes quantum relations, quantum metrics, and so on, it is still the simple and obvious choice.
} 
term quantum graph to mean a reflexive, symmetric quantum relation, i.e., an operator system, as in [3] and [12]; however, the main results of this paper apply to quantum relations generally, and hence also to noncommutative graphs in the broader sense of [11].

The plan of the paper is as follows. We begin in the next section by defining restrictions, pushforwards, and pullbacks in the pure quantum setting; this is done in a simple, concrete fashion, but then in the following section we describe equivalent, conceptually elegant formulations in terms of the notion of "connecting states". These two sections are still fairly introductory.

Next, in Sect. 4, we pass to the general mixed classical/quantum setting, modelled by a unital $*$-subalgebra $\mathcal{M}$ of the $m \times m$ matrix algebra $M_{m}$. We define quantum relations in this more general setting and show how they reduce to ordinary classical relations in the pure classical setting when $\mathcal{M}$ is the diagonal subalgebra of $M_{m}$. Then in Sect. 5 we describe an equivalent abstract characterization of quantum relations framed in terms of connecting states.

Equipped with both concrete and abstract characterizations of quantum relations, we then proceed in the following three sections to define and analyze notions of restriction, pushforward, and pullback in this setting. It will be seen that these notions restrict to those discussed in Sects. 2 and 3 in the pure quantum setting when $\mathcal{M}=M_{m}$, and to the usual classical notions in the pure classical setting.

In an appendix I explain the significance of the mixed classical/quantum setting modelled on a unital $*$-subalgebra of $M_{m}$ in terms of superselection sectors. I also make explicit how the preceding material explains how such things as quantum codes and quantum confusability graphs should be defined in the mixed classical/quantum setting.

\section{Restrictions, Pushforwards, and Pullbacks}

In the quantum relations setting there are natural notions of restriction, pushforward, and pullback. Suppose we are given quantum relations on $M_{m}$ and $M_{n}$, i.e., linear subspaces $\mathcal{V} \subseteq M_{m}$ and $\mathcal{W} \subseteq M_{n}$. If $E$ is any projection in $M_{m}$, meaning that $E=$ $E^{2}=E^{*}$, and $\Phi: M_{m} \rightarrow \bar{M}_{n}$ is a CPTP map expressed as $\Phi(\rho)=\sum_{i=1}^{d} K_{i} \rho K_{i}^{*}$, then we define

- the restriction of $\mathcal{V}$ to $E M_{m} E$ to be $E \mathcal{V} E=\{E A E: A \in \mathcal{V}\}$

- the pushforward of $\mathcal{V}$ along $\Phi$ to be

$$
\begin{aligned}
\overrightarrow{\mathcal{V}} & =\sum K_{i} \mathcal{V} K_{j}^{*} \\
& =\operatorname{span}\left\{K_{i} A K_{j}^{*}: A \in \mathcal{V}, 1 \leq i, j \leq d\right\} \subseteq M_{n}
\end{aligned}
$$

- the pullback of $\mathcal{W}$ along $\Phi$ to be

$$
\begin{aligned}
\overleftarrow{\mathcal{W}} & =\sum K_{i}^{*} \mathcal{W} K_{j} \\
& =\operatorname{span}\left\{K_{i}^{*} B K_{j}: B \in \mathcal{W}, 1 \leq i, j \leq d\right\} \subseteq M_{m}
\end{aligned}
$$


If $\operatorname{rank}(E)=r$ then $E M_{m} E$ can be identified with $M_{r}$, and $E \mathcal{V} E$ with a linear subspace of $M_{r}$, so that the restriction of $\mathcal{V}$ can be regarded as a quantum relation on a smaller space.

These definitions are simple and concrete. It is easy to check that if $\mathcal{V}$ and $\mathcal{W}$ are quantum graphs (i.e., operator systems) then so are $E \mathcal{V} E \subseteq M_{r}, \overrightarrow{\mathcal{V}} \subseteq M_{n}$, and $\overleftarrow{\mathcal{W}} \subseteq M_{m}$. However, the Kraus matrices $K_{i}$ are not uniquely determined by the map $\Phi$ and it is not immediately apparent that the definitions of $\overrightarrow{\mathcal{V}}$ and $\overleftarrow{\mathcal{W}}$ are independent of this choice. The definitions are also rather unmotivated. For instance, when $\mathcal{V}$ is a quantum graph its restriction is to be thought of as analogous to the induced subgraph construction in classical graph theory. But an induced subgraph is obtained by choosing a subset of the vertex set and throwing out all edges which extend out of this subset, whereas our definition of restriction involves compressing everything in $\mathcal{V}$ to the range of $E$. So the validity of the analogy is unclear.

These concerns will be addressed in the next section, when we discuss how the quantum relations point of view leads to the definitions given above. But first, let us explain how these operations relate to error correction.

Consider first the classical setting in which a channel is modelled by a probabilistic transition from an initial set of states $S$ to a final set of states $T$. That is, each initial state has some (possibly zero) probability of going to each of the final states. Such a transition is represented by a stochastic matrix. The confusability graph is specified by placing an edge between two initial states if there exists a final state to which they each have a nonzero probability of transitioning.

As I mentioned earlier, since each initial state can certainly end up at the same state as itself, it is natural to include a loop at each vertex in this graph. A code in this classical setting is then an independent subset of $S$, i.e., a set of vertices with the property that the induced subgraph contains only loops, with no edges between distinct vertices. In the terminology of Sect. 1, the induced subgraph is diagonal. The quantum analog of this would be a projection $E$ with the property that the induced quantum subgraph $E \mathcal{V} E$ is diagonal, i.e., $E \mathcal{V} E=\mathbb{C} \cdot E$. If $\mathcal{V}_{\Phi}$ is the quantum confusability graph mentioned in Sect. 1, this statement exactly expresses the Knill-Laflamme error correction conditions [6]. So

the statement that the range of $E$ is a quantum code is equivalent to the statement that E induces a diagonal quantum subgraph, just as in the classical case a code is a subset of the confusability graph for which the induced subgraph is diagonal.

A more sophisticated way to specify the classical confusability graph for a probabilistic transition from $S$ to $T$ is to say that it is the pullback of the diagonal relation on $T$. Here we define the pullback to $S$ of a graph on $T$ by putting an edge between two elements of $S$ if they have a nonzero probability of mapping to adjacent elements of $T$. The quantum analog of this construction would be the pullback along a CPTP map $\Phi: M_{m} \rightarrow M_{n}$ of the diagonal quantum relation on $M_{n}$. According to the definition of quantum pullback given above, this would be

$$
\operatorname{span}\left\{K_{i}^{*} K_{j}: 1 \leq i, j \leq d\right\} \subseteq M_{m},
$$


which is exactly the quantum confusability graph $\mathcal{V}_{\Phi}$. That is,

the quantum confusability graph $\mathcal{V}_{\Phi}$ associated to a CPTP map $\Phi: M_{m} \rightarrow M_{n}$ is the pullback along $\Phi$ of the diagonal quantum relation on $M_{n}$, just as the clasical confusability graph associated to a classical channel is the pullback of the diagonal relation.

The passage of a message through sequential channels provides a simple illustration of the value of the pullback construction. Suppose we are given classical channels from $S$ to $T$ and from $T$ to $U$. Then their composition defines a channel from $S$ to $U$, and the confusability graph of this composition is the pullback to $S$ of the confusability graph for the $T$-to- $U$ channel. In other words, it is the pullback of the pullback of the diagonal relation on $U$. The same statement can be made in the quantum setting, as one can see by a short computation.

A similar construction is the pushforward of the diagonal relation on $S$. This "dual" confusability graph classically includes an edge between two states in $T$ if they might have originated in the same state of $S$. It might be used by the recipient of a signal which was sent through a noisy channel without the aid of a code, as a way to keep track of possible ambiguity. (This could also be a model of a noisy measurement process in which nature is the "sender".) The quantum analog would simply be the quantum pushforward of the diagonal quantum relation.

Pushforwards and pullbacks give rise to notions of "morphism". Namely, we may regard $\Phi$ as a morphism from $\mathcal{V}$ to $\mathcal{W}$ if $\overrightarrow{\mathcal{V}} \subseteq \mathcal{W}$, or, alternatively, if $\mathcal{V} \subseteq \overleftarrow{\mathcal{W}}$. These two conditions are not equivalent, even in the classical case: the classical analog of the first says that any possible targets of two adjacent vertices in $S$ must be adjacent in $T$, while the second says that any two adjacent vertices in $S$ must have some possible targets which are adjacent in $T$. The quantum version of the first, stronger, condition is identical to Stahlke's notion of "noncommutative graph homomorphism" described in [11]:

a CPTP map $\Phi: M_{m} \rightarrow M_{n}$ is a "noncommutative graph homomorphism"

[11] between operator systems $\mathcal{V} \subseteq M_{m}$ and $\mathcal{W} \subseteq M_{n}$ if $\overrightarrow{\mathcal{V}} \subseteq \mathcal{W}$.

\section{Connecting States}

Now let us see why the definitions of restrictions, pushforwards, and pullbacks given above are natural. The idea is to think of elements of an operator space as "connecting" states. We could say that two pure states $|\alpha\rangle,|\beta\rangle \in \mathbb{C}^{m}$ are connected by a quantum relation $\mathcal{V} \subseteq M_{m}$ if $\langle\alpha|B| \beta\rangle \neq 0$ for some $B \in \mathcal{V}$. However, quantum relations are not determined by this kind of information. For example, take $\mathcal{V}_{1}$ to be the set of $2 \times 2$ matrices of the form $\left[\begin{array}{ll}a & b \\ c & a\end{array}\right]$ with $a, b, c \in \mathbb{C}$ and take $\mathcal{V}_{2}$ to be the full $2 \times 2$ matrix algebra $M_{2}$. These are both quantum graphs on $M_{2}$, i.e., operator systems. It is routine to check that $|\alpha\rangle,|\beta\rangle \in \mathbb{C}^{2}$ are connected by $\mathcal{V}_{1}$ if and only if neither of them is the zero vector if and only if they are connected by $\mathcal{V}_{2}$. Thus, $\mathcal{V}_{1}$ and $\mathcal{V}_{2}$ are distinct quantum relations which connect the same pairs of states in $\mathbb{C}^{2}$. 
We must instead consider states not in $\mathbb{C}^{m}$ but in $\mathbb{C}^{m} \otimes \mathbb{C}^{k} \cong \mathbb{C}^{m k}$. That is, we consider states of a composite system formed from the original system and some other system. Then we can define $|\alpha\rangle,|\beta\rangle \in \mathbb{C}^{m k}$ to be connected by $\mathcal{V}$ if there exists $B \in \mathcal{V}$ such that

$$
\left\langle\alpha\left|\left(B \otimes I_{k}\right)\right| \beta\right\rangle \neq 0 .
$$

It is not hard to show that $\mathcal{V}$ is indeed determined by which pairs of states it connects in $\mathbb{C}^{m k}$ for arbitrary $k$; indeed, $k=m$ suffices. See Lemma 5.2 below.

It is convenient to also consider mixed states. First of all, observe that

$$
|\alpha\rangle\left\langle\alpha\left|\left(B \otimes I_{k}\right)\right| \beta\right\rangle\langle\beta|
$$

is nonzero if and only if the scalar factor $\left\langle\alpha\left|\left(B \otimes I_{k}\right)\right| \beta\right\rangle$ is nonzero. So we can also say that $\mathcal{V}$ connects $|\alpha\rangle$ and $|\beta\rangle$ if and only if the preceding expresion is nonzero for some $B \in \mathcal{V}$. More generally, say that $\mathcal{V}$ connects positive matrices $A, C \in M_{m k} \cong$ $M_{m} \otimes M_{k}$ with unit trace if

$$
A\left(B \otimes I_{k}\right) C \neq 0
$$

for some $B \in \mathcal{V}$.

Since $\mathcal{V}$ is already determined by the pairs of (composite) pure states that it connects, it is certainly determined by the pairs of mixed states that it connects. Any positive matrix can be expressed as a sum of positive, orthogonal rank one matrices, so there is little difference between the two characterizations.

This point of view makes the constructions described in the last section transparent. Let $k$ be a natural number, let $\mathcal{V} \subseteq M_{m}$ and $\mathcal{W} \subseteq M_{n}$ be quantum relations, let $E \in M_{m}$ be a rank $r$ projection, and let $\Phi: M_{m} \rightarrow M_{n}$ be a CPTP map. Then

- $E \mathcal{V} E$ connects mixed states $A, C \in E M_{m} E \otimes M_{k} \cong M_{r} \otimes M_{k}$ if and only if $\mathcal{V}$ connects them

- $\overrightarrow{\mathcal{V}}$ connects mixed states $A, C \in M_{n} \otimes M_{k}$ if and only if $\mathcal{V}$ connects $\Phi^{*}(A)$ and $\Phi^{*}(C)$

- $\overleftarrow{\mathcal{W}}$ connects mixed states $A, C \in M_{m} \otimes M_{k}$ if and only if $\mathcal{W}$ connects $\Phi(A)$ and $\Phi(C)$.

See Proposition 6.2, Theorem 7.4, and Theorem 8.2 below.

Informally, the mixed states that $E \mathcal{V} E$ connects are just the mixed states that live on $E$ and are connected by $\mathcal{V}$. This jibes better with the "induced subgraph" intuition: in order to restrict $\mathcal{V}$ to $E$, look at the pairs of states that are connected by $\mathcal{V}$, and throw out any of them which do not lie under $E$.

Pushforward and pullback are also easily understood in terms of connection. The states connected by $\overrightarrow{\mathcal{V}}$ are just the states whose images under $\Phi^{*}$ are connected by $\mathcal{V}$, and the states connected by $\overleftarrow{\mathcal{W}}$ are just the states whose images under $\Phi$ are connected by $\mathcal{W}$. This characterization shows that the definitions of pushforward and pullback only depend on the map $\Phi$, not the choice of Kraus matrices. 
The whole point of the noncommutative confusability graph is that it connects mixed states $A$ and $C$ if and only if $\Phi(A) \Phi(C) \neq 0$, i.e., their image states could be confused. That is the same as saying that their image states are connected by the diagonal quantum relation.

We can also use the idea of connecting mixed states to give an intrinsic characterization of the "noncommutative (directed) bipartite graphs" of Duan, Severini, and Winter [3]. Given a CPTP map $\Phi: M_{m} \rightarrow M_{n}$ with Kraus matrices $K_{i}$, they defined this to be the span of the matrices $K_{i}$. This span is no longer an operator system in general, but it is still an operator space and hence still counts, in our terminology, as a quantum relation. Its intrinsic characterization is simple: if $\mathcal{V}=\operatorname{span}\left\{K_{i}\right\} \subseteq M_{n, m}$, then for any mixed states $A \in M_{m} \otimes M_{k}$ and $C \in M_{n} \otimes M_{k}$, we have $C\left(B \otimes I_{k}\right) A \neq 0$ for some $B \in \mathcal{V}$ if and only if $\Phi(A) C \neq 0$ (letting $\Phi$ act entrywise on matrices in $\left.M_{m} \otimes M_{k} \cong M_{k}\left(M_{m}\right)\right)$. That is,

the noncommutative bipartite graph associated to a CPTP map $\Phi: M_{m} \rightarrow M_{n}$ connects mixed states $A \in M_{m} \otimes M_{k}$ and $C \in M_{n} \otimes M_{k}$ if and only if $\Phi(A) C \neq$ 0, i.e., there is a possibility of confusing the image of $A$ with $C$.

One direction is trivial: if $C\left(B \otimes I_{k}\right) A=0$ for all $B \in \mathcal{V}$, then in particular $C\left(K_{i} \otimes\right.$ $\left.I_{k}\right) A=0$ for all $i$; multiplying on the right by $\left(K_{i}^{*} \otimes I_{k}\right)$ and summing over $i$ then yields $C \Phi(A)=0$. This is equivalent to $\Phi(A) C=0$ since $\Phi(A)$ and $C$ are positive. The reverse direction follows from Lemma 7.3 (cf. the proof of Theorem 7.4).

\section{General Quantum Relations}

The definition of "quantum relations" given in [12] was more general than the one described above and actually encompasses both the classical and quantum cases. By placing the notions of channel, confusability graph, code, etc., in this context we obtain a common generalization in which the classical and quantum cases are not merely analogous, but literally special cases of a single theory. This material will be presented more formally, with proofs of most results.

Let $\mathcal{M}$ be a unital $*$-subalgebra of $M_{m}$. (In [12] it could be an arbitrary von Neumann algebra in infinite dimensions.) The two most important cases to keep in mind are $\mathcal{M}=M_{m}$, the full matrix algebra, and $\mathcal{M}=D_{m}$, the subalgebra of diagonal matrices. However, other cases could arise in the presence of superselection rules; see the appendix.

Let

$$
\mathcal{M}^{\prime}=\left\{B \in M_{m}: A B=B A \text { for all } A \in \mathcal{M}\right\}
$$

be the commutant of $\mathcal{M}$. The commutant of $M_{m}$ is the scalar algebra $\mathbb{C} \cdot I_{m}$, and the commutant of $D_{m}$ is itself. Von Neumann's double commutant theorem states that $\mathcal{M}^{\prime \prime}=\mathcal{M}$ always holds.

Definition 4.1 ([12], Definition 2.1) A quantum relation on $\mathcal{M}$ is an $\mathcal{M}^{\prime}-\mathcal{M}^{\prime}$ bimodule, i.e., a linear subspace $\mathcal{V} \subseteq M_{m}$ satisfying $\mathcal{M}^{\prime} \mathcal{V} \mathcal{M}^{\prime} \subseteq \mathcal{V}$. 
Here we use the operator space product $\mathcal{V} \mathcal{W}=\operatorname{span}\{A B: A \in \mathcal{V}, B \in \mathcal{W}\}$.

Definition 4.2 ([12], Definition 2.4) $\mathcal{M}^{\prime}$ is the diagonal quantum relation on $\mathcal{M}$. A quantum relation $\mathcal{V}$ is

- reflexive if $\mathcal{M}^{\prime} \subseteq \mathcal{V}$

- symmetric if $\mathcal{V}^{*}=\mathcal{V}$

- antisymmetric if $\mathcal{V} \cap \mathcal{V}^{*} \subseteq \mathcal{M}^{\prime}$

- transitive if $\mathcal{V}^{2} \subseteq \mathcal{V}$.

If $\mathcal{M}=M_{m}$ then $\mathcal{M}^{\prime}$ is just the set of scalar matrices, $\mathcal{M}^{\prime}=\mathbb{C} \cdot I_{m}$, and so any linear subspace of $M_{m}$ counts as a quantum relation on $M_{m}$ according to Definition 4.1. At the other extreme, it is not hard to check that quantum relations on $D_{m}$ have a very transparent form. Let $E_{i j}$ be the $m \times m$ matrix with a 1 in the $(i, j)$ entry and 0 's elsewhere.

Proposition 4.3 ([12], Proposition 2.2) If $R$ is any subset of $\{(i, j): 1 \leq i, j \leq m\}$ then

$$
\mathcal{V}_{R}=\operatorname{span}\left\{E_{i j}:(i, j) \in R\right\} \subseteq M_{m}
$$

is a quantum relation on $D_{m}$, i.e., a $D_{m}-D_{m}$ bimodule, and every quantum relation on $D_{m}$ equals $\mathcal{V}_{R}$ for some $R$. This establishes a 1-1 correspondence between the classical relations on the set $\{1, \ldots, m\}$ and the quantum relations on $D_{m}$.

This simple result explains the justification for the term "quantum relation" and also shows the value of letting $\mathcal{M}$ be any unital $*$-subalgebra of $M_{m}$, not just $M_{m}$ itself. By taking this step we produce a common generalization of both the classical $\left(\mathcal{M}=D_{m}\right)$ and elementary quantum $\left(\mathcal{M}=M_{m}\right)$ cases. The following result shows that the terminology of Definition 4.2 legitimately generalizes the classical case.

Proposition 4.4 ([12], Proposition 2.5) In the notation of Proposition 4.3, the diagonal quantum relation on $D_{m}$ is $\mathcal{V}_{\Delta}$ where $\Delta=\{(i, i): 1 \leq i \leq m\}$. A classical relation $R$ on $\{1, \ldots, m\}$ is reflexive, symmetric, antisymmetric, or transitive in the ordinary sense if and only if the quantum relation $\mathcal{V}_{R}$ has the same property in the sense of Definition 4.2.

The proof is easy.

Earlier we interpreted classical graphs as sets equipped with reflexive, symmetric relations, and defined quantum graphs to be operator systems. Both notions are subsumed in the following definition.

Definition 4.5 ([12], Definition 2.6 (d)) A quantum graph on $\mathcal{M}$ is a reflexive, symmetric quantum relation on $\mathcal{M}$.

In the case $\mathcal{M}=M_{m}$, this would just mean an operator system in $M_{m}$; in the case $\mathcal{M}=D_{m}$, by Propositions 4.3 and 4.4 it effectively becomes a classical reflexive, symmetric relation on a set. 


\section{Intrinsic Characterization}

The definition of a quantum relation on a unital $*$-subalgebra $\mathcal{M} \subseteq M_{m}$ given in Definition 4.1 appears to depend on the representation of $\mathcal{M}$, i.e., on the value of $m$ and perhaps also on the way $\mathcal{M}$, regarded as an abstract algebraic structure, is situated in $M_{m}$. However, this definition is in fact effectively representation-independent, in the following sense. Say that $*$-algebras $\mathcal{M}$ and $\mathcal{N}$ are $*$-isomorphic if there is a linear bijection between them that is compatible with the product and adjoint operations.

Proposition 5.1 ([12], Theorem 2.7) Let $\mathcal{M} \subseteq M_{m}$ and $\mathcal{N} \subseteq M_{n}$ be unital *-subalgebras and suppose they are *-isomorphic. Then there is a natural 1-1 correspondence between the quantum relations on $\mathcal{M}$ and the quantum relations on $\mathcal{N}$. This correspondence takes the diagonal quantum relation on $\mathcal{M}$ to the diagonal quantum relation on $\mathcal{N}$ and is compatible with the operator space product and adjoint operations on quantum relations.

To see how the correspondence works, consider the case where $n=m k$ and $\mathcal{N}=$ $\mathcal{M} \otimes I_{k} \subseteq M_{m} \otimes M_{k} \cong M_{n}$. Then $\mathcal{N}^{\prime}=\mathcal{M}^{\prime} \otimes M_{k}$ and, identifying $M_{n}$ with $M_{m} \otimes M_{k}$, the bimodules over $\mathcal{N}^{\prime}$ in $M_{n}$ are precisely the sets of the form $\mathcal{V} \otimes M_{k}$ for $\mathcal{V}$ a bimodule over $\mathcal{M}^{\prime}$ in $M_{m}$. The full result of Proposition 5.1 is not much harder than this special case because arbitrary $*$-isomorphisms between von Neumann algebras are not much more general than this.

Thus, the notion of a quantum relation on $\mathcal{M}$ is effectively independent of the representation of $\mathcal{M}$. We therefore expect that there should be an "intrinsic" characterization of them which does not reference the ambient matrix algebra. This can be achieved using the idea of connecting states introduced in Sect. 3.

At that point it was convenient to consider mixed states, since we wanted to push forward and pull back along a CPTP map, which can convert pure states to mixed states. For the purpose of abstract characterization, it is better to generalize pure states, which can be identified with rank one projections, to projections of arbitrary rank. A direct connection between the two approaches can be made by observing that for any positive matrices $A, C \in M_{m} \otimes M_{k}$, we have $A\left(B \otimes I_{k}\right) C \neq 0$ if and only if $[A]\left(B \otimes I_{k}\right)[C] \neq 0$, where $[A]$ denotes the range projection of $A$, i.e., the orthogonal projection onto the range of $A$.

Proposition 5.2 ([12], Lemma 2.8) Let $\mathcal{V}$ be a proper subspace of $M_{m}$ and let $B \in$ $M_{m} \backslash \mathcal{V}$.

(a) There exists a natural number $k$ and vectors $|\alpha\rangle,|\beta\rangle \in \mathbb{C}^{m} \otimes \mathbb{C}^{k}$ such that

$$
\left\langle\alpha\left|\left(A \otimes I_{k}\right)\right| \beta\right\rangle=0 \quad \text { for all } A \in \mathcal{V}
$$

but

$$
\left\langle\alpha\left|\left(B \otimes I_{k}\right)\right| \beta\right\rangle \neq 0 .
$$


(b) If $\mathcal{M}$ is a unital $*$-subalgebra of $M_{m}$ and $\mathcal{V}$ is a quantum relation on $\mathcal{M}$, then there exist $k \in \mathbb{N}$ and projections $P, Q \in M_{k}(\mathcal{M})$ such that

$$
P\left(A \otimes I_{k}\right) Q=0 \quad \text { for all } A \in \mathcal{V}
$$

but

$$
P\left(B \otimes I_{k}\right) Q \neq 0 \text {. }
$$

Proof (a) We prove the result with $k=m$. First, observe that $M_{m}$ becomes an inner product space when equipped with the Hilbert-Schmidt inner product

$$
\left\langle A_{1}, A_{2}\right\rangle=\operatorname{Tr}\left(A_{1}^{*} A_{2}\right) .
$$

So by elementary facts about inner product spaces, since $\mathcal{V}$ is a subspace and $B \notin \mathcal{V}$, there must exist $C \in M_{m}$ satisfying

$$
\operatorname{Tr}(A C)=0 \quad \text { for all } A \in \mathcal{V}
$$

but

$$
\operatorname{Tr}(B C) \neq 0 .
$$

Let $\left|c_{1}\right\rangle, \ldots,\left|c_{m}\right\rangle \in \mathbb{C}^{m}$ be the columns of $C$, let $\left|e_{1}\right\rangle, \ldots,\left|e_{m}\right\rangle$ be the standard basis vectors in $\mathbb{C}^{m}$, and let $|\alpha\rangle,|\beta\rangle \in \mathbb{C}^{m} \otimes \mathbb{C}^{m} \cong \mathbb{C}^{m} \oplus \cdots \oplus \mathbb{C}^{m}$ be the vectors

$$
|\alpha\rangle=\left|e_{1}\right\rangle \oplus \cdots \oplus\left|e_{m}\right\rangle \quad|\beta\rangle=\left|c_{1}\right\rangle \oplus \cdots \oplus\left|c_{m}\right\rangle
$$

Then

$$
\left\langle\alpha\left|\left(A \otimes I_{m}\right)\right| \beta\right\rangle=\sum_{i}\left\langle e_{i}|A| c_{i}\right\rangle=\operatorname{Tr}(A C)=0
$$

for all $A \in \mathcal{V}$, and similarly $\left\langle\alpha\left|\left(B \otimes I_{m}\right)\right| \beta\right\rangle=\operatorname{Tr}(B C) \neq 0$.

(b) Find $|\alpha\rangle,|\beta\rangle \in \mathbb{C}^{m} \otimes \mathbb{C}^{m}$ as in part (a) and let $P, Q \in M_{m} \otimes M_{m}$ be the orthogonal projections onto $\left(\mathcal{M}^{\prime} \otimes I_{m}\right)|\alpha\rangle=\left\{\left(A \otimes I_{m}\right)|\alpha\rangle: A \in \mathcal{M}^{\prime}\right\}$ and $\left(\mathcal{M}^{\prime} \otimes\right.$ $\left.I_{m}\right)|\beta\rangle=\left\{\left(A \otimes I_{m}\right)|\beta\rangle: A \in \mathcal{M}^{\prime}\right\}$, respectively. Since these spaces are invariant for the $*$-algebra $\mathcal{M}^{\prime} \otimes I_{m}, P$ and $Q$ belong to its commutant $\left(\mathcal{M}^{\prime} \otimes I_{m}\right)^{\prime}=\mathcal{M} \otimes M_{m} \cong$ $M_{m}(\mathcal{M})$.

Now $Q|\beta\rangle=|\beta\rangle$, so the range of $\left(B \otimes I_{m}\right) Q$ contains the vector $\left(B \otimes I_{m}\right)|\beta\rangle$, which is not orthogonal to $|\alpha\rangle$. Since $|\alpha\rangle$ belongs to the range of $P$, it follows that $P\left(B \otimes I_{m}\right) Q \neq 0$. However, if $A \in \mathcal{V}$ and $A_{1}, A_{2} \in \mathcal{M}^{\prime}$ then $A_{1}^{*} A A_{2} \in \mathcal{V}$, so that

$$
\left\langle\left(A_{1} \otimes I_{m}\right) \alpha\left|\left(A \otimes I_{m}\right)\right|\left(A_{2} \otimes I_{m}\right) \beta\right\rangle=\left\langle\alpha\left|\left(A_{1}^{*} A A_{2} \otimes I_{m}\right)\right| \beta\right\rangle=0 .
$$

Since this is true for any $A_{1}, A_{2} \in \mathcal{M}^{\prime}$, it follows that $\left\langle\alpha^{\prime}\left|\left(A \otimes I_{m}\right)\right| \beta^{\prime}\right\rangle=0$ for all $\left|\alpha^{\prime}\right\rangle$ and $\left|\beta^{\prime}\right\rangle$ in the ranges of $P$ and $Q$, respectively. Thus $P\left(A \otimes I_{m}\right) Q=0$. 
Say that $\mathcal{V}$ connects projections $P, Q \in M_{k}(\mathcal{M})$ if there exists $A \in \mathcal{V}$ such that $P\left(A \otimes I_{k}\right) Q \neq 0$. The preceding result shows that $\mathcal{V}$ is determined by the pairs of projections it connects in this manner: we can tell whether a given $B \in M_{m}$ belongs to $\mathcal{V}$ by testing whether it connects any pair of projections that is not connected by $\mathcal{V}$. Since this is a crucial point, let us emphasize it: if $\mathcal{M}$ is a unital $*$-subalgebra of $M_{m}$ then an $\mathcal{M}^{\prime}-\mathcal{M}^{\prime}$ bimodule is determined by the pairs of projections in $M_{m}(\mathcal{M})$ that it connects.

In fact, quantum relations can be characterized abstractly in these terms. We give the relevant definition first, and then state the equivalence with Definition 4.1 as a theorem. To avoid confusion, we will now refer to quantum relations in the sense of Definition 4.1 as concrete quantum relations.

Let $\mathcal{P}\left(M_{k}(\mathcal{M})\right)$ denote the set of projections in $M_{k}(\mathcal{M})$, given the topology it inherits from $M_{k}(\mathcal{M})$.

Definition 5.3 ([12], Definition 2.24) Let $\mathcal{M}$ be a unital $*$-subalgebra of $M_{m}$, and for each $k \in \mathbb{N}$ let $\mathcal{R}_{k}$ be an open subset of $\mathcal{P}\left(M_{k}(\mathcal{M})\right) \times \mathcal{P}\left(M_{k}(\mathcal{M})\right)$. Then the sequence $\left(\mathcal{R}_{k}\right)$ is an intrinsic quantum relation if

(i) $(0,0) \notin \mathcal{R}_{k}$

(ii) $\left(\bigvee P_{i}, \bigvee Q_{j}\right) \in \mathcal{R}_{k}$ if and only if $\left(P_{i_{0}}, Q_{j_{0}}\right) \in \mathcal{R}_{k}$ for some $i_{0}, j_{0}$

(iii) $(P,[B Q]) \in \mathcal{R}_{k}$ if and only if $\left(\left[B^{*} P\right], Q\right) \in \mathcal{R}_{l}$

for all $k, l \in \mathbb{N}$, all projections $P, P_{i}, Q_{j} \in M_{k}(\mathcal{M})$ and $Q \in M_{l}(\mathcal{M})$, and all scalar matrices $B \in I_{m} \otimes M_{k, l}$.

In condition (ii) the join $\bigvee P_{i}$ of a finite family of projections $\left(P_{i}\right)$ is defined to be the orthogonal projection onto the span of their ranges. In (iii), recall that the notation $[B]$ indicates the range projection of $B$.

$\mathcal{R}_{k}$ is to be thought of as the pairs of projections in $M_{k}(\mathcal{M})$ which are connected by some concrete quantum relation $\mathcal{V} \subseteq M_{m}$. To say that each $\mathcal{R}_{k}$ is open is to say that if two projections are connected then so are any two projections sufficiently close to them. Condition (i) is trivial, condition (ii) is the basic axiom characterizing connection, and condition (iii) is a statement about scalar compatibility that is typical of what one sees when working at matrix levels. The point is that if $B$ is a scalar matrix then

$$
\begin{aligned}
P\left(A \otimes I_{k}\right)[B Q]=0 & \Leftrightarrow P\left(A \otimes I_{k}\right) B Q=0 \\
& \Leftrightarrow P B\left(A \otimes I_{l}\right) Q=0 \\
& \Leftrightarrow\left[B^{*} P\right]\left(A \otimes I_{l}\right) Q=0,
\end{aligned}
$$

since $\left(A \otimes I_{k}\right) B=B\left(A \otimes I_{l}\right)$.

Proposition 5.2 (b) shows us how to go from concrete quantum relations, as characterized by Definition 4.1, to intrinsic quantum relations, axiomatized as in Definition 5.3. Namely, given $\mathcal{V}$, for each $k \in \mathbb{N}$ let $\mathcal{R}_{k}$ be the set of pairs $(P, Q)$ of projections in $M_{k}(\mathcal{M})$ such that $P\left(A \otimes I_{k}\right) Q \neq 0$ for some $A \in \mathcal{V}$. Conversely, given an intrinsic quantum relation $\left(\mathcal{R}_{k}\right)$ one recovers the concrete quantum relation that corresponds 
to it as the set of $A \in M_{m}$ satisfying

$$
P\left(A \otimes I_{k}\right) Q=0
$$

for all $k \in \mathbb{N}$ and all $(P, Q) \notin \mathcal{R}_{k}$, i.e., the set of matrices which do not connect any pair of projections they are not supposed to connect.

Theorem 5.4 ([12], Theorem 2.32) For any unital $*$-subalgebra $\mathcal{M}$ of $M_{m}$, the two constructions just described establish a 1-1 correspondence between the concrete and intrinsic quantum relations on $\mathcal{M}$.

The proof of Theorem 5.4 is somewhat complicated.

Observe that the characterization of quantum relations provided by Definition 5.3 is "intrinsic" to $\mathcal{M}$ in the sense that it makes no reference to the ambient matrix algebra in which $\mathcal{M}$ is located. It is manifestly compatible with $*$-isomorphisms.

\section{Restrictions}

Theorem 5.4 allows us to pass back and forth between concrete and intrinsic quantum relations, and we will do this repeatedly in the sequel.

An $\mathcal{M}^{\prime}-\mathcal{M}^{\prime}$ bimodule is a straightforward object, especially when $\mathcal{M}=M_{m}$ and $\mathcal{M}^{\prime}=\mathbb{C} \cdot I_{m}$. The value in having a more complicated intrinsic characterization in terms of connecting projections is that some constructions are more naturally understood in these terms. For instance, the natural notion of "subobject" is the following.

Definition 6.1 Let $\mathcal{M}$ be a unital $*$-subalgebra of $M_{m}$, let $\left(\mathcal{R}_{k}\right)$ be an intrinsic quantum relation on $\mathcal{M}$, and let $E \in \mathcal{M}$ be a projection of rank $r$. The restriction of $\left(\mathcal{R}_{k}\right)$ to $E \mathcal{M} E \subseteq E M_{m} E \cong M_{r}$ is the intrinsic quantum relation $\left(\tilde{\mathcal{R}}_{k}\right)$ on $E \mathcal{M} E$ defined by setting

$$
\tilde{\mathcal{R}}_{k}=\left\{(P, Q) \in \mathcal{R}_{k}: P, Q \leq E \otimes I_{k}\right\}
$$

for all $k \in \mathbb{N}$.

It is straightforward to verify that $\left(\tilde{\mathcal{R}}_{k}\right)$ as defined above has the properties of an intrinsic quantum relation described in Definition 5.3. So according to Theorem 5.4, if $\left(\mathcal{R}_{k}\right)$ is associated to the concrete quantum relation $\mathcal{V} \subseteq M_{m}$, its restriction $\left(\tilde{\mathcal{R}}_{k}\right)$ must be associated to a concrete quantum relation $\tilde{\mathcal{V}} \subseteq M_{r}$ on $E \mathcal{M} E$. This concrete restriction has a simple direct characterization:

Proposition 6.2 Let $\mathcal{V}$ be a concrete quantum relation on $\mathcal{M} \subseteq M_{m}$ and let $E \in \mathcal{M}$ be a projection. Then the restriction $\tilde{\mathcal{V}}$ of $\mathcal{V}$ to $E \mathcal{M} E$ is concretely given as $\tilde{\mathcal{V}}=E \mathcal{V} E$.

Proof First observe that the commutant of $E \mathcal{M} E$ in $E M_{m} E \cong M_{r}$ is $E \mathcal{M}^{\prime} E$. For the sake of completeness, the standard proof goes as follows. The containment $E \mathcal{M}^{\prime} E \subseteq$ $(E \mathcal{M} E)^{\prime}$ is clear because

$$
(E B E)(E A E)=E B A E=E A B E=(E A E)(E B E)
$$


for all $A \in \mathcal{M}$ and $B \in \mathcal{M}^{\prime}$, showing that everything in $E \mathcal{M}^{\prime} E$ commutes with everything in $E \mathcal{M} E$. For the reverse containment, by the double commutant theorem it suffices to show that $\left(E \mathcal{M}^{\prime} E\right)^{\prime} \subseteq E \mathcal{M} E$. So let $A \in M_{r} \cong E M_{m} E$ belong to the commutant of $E \mathcal{M}^{\prime} E$. Regarding $A$ as an element of $M_{m}$ satisfying $A=E A E$, this means that $(E B E) A=A(E B E)$ for all $B \in \mathcal{M}^{\prime}$. But $B E=E B$ and $A E=$ $E A=A$, so it follows that $B A=A B$ for all $B \in \mathcal{M}^{\prime}$, i.e., by the double commutant theorem, $A \in \mathcal{M}$. Thus $A \in E \mathcal{M} E$, as desired.

The computations

$$
(E A E)(E B E)=E A B E \quad \text { and } \quad(E B E)(E A E)=E B A E
$$

for $A \in \mathcal{V}$ and $B \in \mathcal{M}^{\prime}$ now show that $E \mathcal{V} E$ is a bimodule over $(E \mathcal{M} E)^{\prime}=E \mathcal{M}^{\prime} E$, i.e., it is a quantum relation on $E \mathcal{M} E$.

Now let $\left(\mathcal{R}_{k}\right)$ be the intrinsic quantum relation on $\mathcal{M}$ corresponding to $\mathcal{V},\left(\tilde{\mathcal{R}}_{k}\right)$ the restriction of $\left(\mathcal{R}_{k}\right)$ to $E \mathcal{M} E$ according to Definition 6.1, and $\tilde{\mathcal{R}}_{k}^{\prime}$ the intrinsic quantum relation on $E \mathcal{M} E$ corresponding to $E \mathcal{V} E$. We must show that $\left(\tilde{\mathcal{R}}_{k}\right)=\left(\tilde{\mathcal{R}}_{k}^{\prime}\right)$.

Fix $k \in \mathbb{N}$. In one direction, if $(P, Q) \in \tilde{\mathcal{R}}_{k}^{\prime}$ then there exists $E A E \in E \mathcal{V} E$ such that

$$
P\left(E A E \otimes I_{k}\right) Q \neq 0 \text {. }
$$

But since $P, Q \leq E \otimes I_{k}$ and

$$
E A E \otimes I_{k}=\left(E \otimes I_{k}\right)\left(A \otimes I_{k}\right)\left(E \otimes I_{k}\right)
$$

this implies that $P\left(A \otimes I_{k}\right) Q \neq 0$, so that $(P, Q)$ belongs to $\mathcal{R}_{k}$ and therefore to $\tilde{\mathcal{R}}_{k}$. Conversely, if $(P, Q) \in \tilde{\mathcal{R}}_{k}$ then $(P, Q) \in \mathcal{R}_{k}$ and so $P\left(A \otimes I_{k}\right) Q \neq 0$ for some $A \in \mathcal{V}$. But since $P, Q \leq E \otimes I_{k}$, this implies that $P\left(E A E \otimes I_{k}\right) Q \neq 0$, and we therefore have $(P, Q) \in \tilde{\mathcal{R}}_{k}^{\prime}$. This completes the proof of the desired equality.

Although this concrete description of the restriction of $\mathcal{V}$ to $E \mathcal{M} E$ is very simple, it is the intrinsic formulation given in Definition 6.1 which brings out its role as a "restriction".

The following definition now becomes natural.

Definition 6.3 Let $\mathcal{V}$ be a quantum graph (a reflexive, symmetric quantum relation) on $\mathcal{M} \subseteq M_{m}$ and let $E \in \mathcal{M}$ be a projection. Then $E$ is independent if the restriction of $\mathcal{V}$ to $E \mathcal{M} E$ is the diagonal quantum relation on $E \mathcal{M} E$.

In the case $\mathcal{M}=D_{m}$, the projection $E$ corresponds to a subset of $\{1, \ldots, m\}$, and $E$ is independent in the above sense if and only if the classical graph corresponding to $\mathcal{V}$ has no nontrivial edges in this subset. That is, Definition 6.3 generalizes the classical notion of an independent subset of a graph. In the case $\mathcal{M}=M_{m}$, the independence condition simply states that

$$
E \mathcal{V} E=\mathbb{C} \cdot E,
$$


which, as we noted earlier, expresses the Knill-Laflamme error correction conditions. So the notion of independence yields a common generalization of classical and quantum codes.

\section{Pushforwards}

Classically, if $f: X \rightarrow Y$ is a function between sets then we can push any relation $R$ on $X$ forward to a relation on $Y$, namely, the relation $\{(f(x), f(y)):(x, y) \in R\}$. Similarly, any relation $\mathcal{R}^{\prime}$ on $Y$ can be pulled back to the relation $\{(x, y):(f(x), f(y)) \in$ $\mathcal{R}^{\prime}$ \} on $X$. We now seek quantum versions of these constructions.

The first point to make is that the classical analog of a quantum channel is not an actual function between sets, but a classical channel which maps points in the domain to probability distributions in the range (representing the likelihood of the given input state being received as various output states). In this context the pushforward of a relation $R$ on $X$ would consist of the pairs $\left(x^{\prime}, y^{\prime}\right) \in Y^{2}$ such that there exists a pair $(x, y) \in R$ for which the transition probabilities $x \rightarrow x^{\prime}$ and $y \rightarrow y^{\prime}$ are both nonzero. The pullback of a relation $S$ on $Y$ would consist of the pairs $(x, y) \in X^{2}$ such that there exists a pair $\left(x^{\prime}, y^{\prime}\right) \in S$ for which the transition probabilities $x \rightarrow x^{\prime}$ and $y \rightarrow y^{\prime}$ are both nonzero.

Since we are working with unital $*$-algebras, it is natural to adopt the Heisenberg picture in which algebras of observables transform. Mathematically, this means that instead of the CPTP map $\Phi: \rho \mapsto \sum K_{i} \rho K_{i}^{*}$ from $M_{m}$ to $M_{n}$ mentioned in Sect. 1, which acts on states, we consider the adjoint map $\Psi: A \mapsto \sum K_{i}^{*} A K_{i}$ from $M_{n}$ to $M_{m}$, which acts on observables. The adjoint of a CPTP map is a unital CP (unital completely positive) map. Taking adjoints reverses arrows, so that pushforwards become pullbacks and vice versa; consequently, to maintain consistency with Sect. 2 we will continue to take the CPTP map $\Phi: \mathcal{M} \rightarrow \mathcal{N}$ as primary, even though at this point it becomes less natural. The map $\Phi$ really should be understood as a map from the predual of $\mathcal{M}$ to the predual of $\mathcal{N}$ whose adjoint unital CP map $\Psi=\Phi^{*}$ takes the $*$-algebra $\mathcal{N}$ to the $*$-algebra $\mathcal{M}$, but any finite-dimensional $*$-algebra can be identified with its predual via the pairing $(A, B) \mapsto \operatorname{Tr}(A B)$, so we need not make this distinction.

The unital CP maps which correspond to actual functions between sets are the unital *-homomorphisms, linear maps $\Psi: \mathcal{N} \rightarrow \mathcal{M}$ which preserve the identity and respect the product and adjoint operations. If $\Psi=\Phi^{*}$ is a $*$-homomorphism and $\left(\mathcal{R}_{k}\right)$ is an intrinsic quantum relation on $\mathcal{M}$, there is an obvious way to push forward a quantum relation $\left(\mathcal{R}_{k}\right)$ on $\mathcal{M}$ along $\Phi$ to a quantum relation $\left(\tilde{\mathcal{R}}_{k}\right)$ on $\mathcal{N}$. Namely, for each $k \in \mathbb{N}$ let $\tilde{\mathcal{R}}_{k}$ consist of those pairs of projections $P, Q \in M_{k}(\mathcal{N})$ with the property that $(\Psi(P), \Psi(Q)) \in \mathcal{R}_{k}$. (Here we abuse notation and also denote by $\Psi$ the map from $M_{k}(\mathcal{N})$ to $M_{k}(\mathcal{M})$ which applies $\Psi$ entrywise.) This definition makes sense because the $*$-homomorphism property ensures that $\Psi(P)$ and $\Psi(Q)$ are projections. It is easy to check that the preceding construction does yield an intrinsic quantum relation on $\mathcal{N}$ ([12], Proposition 2.25 (b)).

But we are interested in general CPTP maps, not just those whose adjoint maps are $*$-homomorphisms. The construction just described no longer works because the 
image of a projection under such a map need not be a projection. However, there is a simple solution to this difficulty. Let us consider two positive matrices $A, B \in M_{n}$ to be equivalent if $[A]=[B]$. Since the range of a Hermitian matrix is the orthocomplement of its kernel, this condition could also be stated as $\operatorname{ker}(A)=\operatorname{ker}(B)$. This notion of equivalence is suitable here because whether positive matrices are connected by a quantum relation depends only on their range projections.

Lemma 7.1 Let $\mathcal{M}$ and $\mathcal{N}$ be unital $*$-subalgebras of $M_{m}$ and $M_{n}$, respectively, let $A, B \in \mathcal{N}$ be positive, and let $\Psi: \mathcal{N} \rightarrow \mathcal{M}$ be a $C P$ map. Then $[A]=[B]$ implies $[\Psi(A)]=[\Psi(B)]$.

Proof Recall that the join $P \vee Q$ of two projections $P$ and $Q$ is the orthogonal projection onto the span of their ranges. We first claim that $[A+B]=[A] \vee[B]$. (We are not yet assuming $[A]=[B]$, only that $A$ and $B$ are both positive.) That is, we claim that $\operatorname{ran}(A+B)=\operatorname{ran}(A)+\operatorname{ran}(B)$. The containment $\subseteq$ is clear. Conversely, suppose $|\alpha\rangle \perp \operatorname{ran}(A+B)$, i.e., $|\alpha\rangle \in \operatorname{ker}(A+B)$. Then

$$
0=\langle\alpha|(A+B)| \alpha\rangle=\langle\alpha|A| \alpha\rangle+\langle\alpha|B| \alpha\rangle
$$

Since $A$ and $B$ are positive, this implies that $\langle\alpha|A| \alpha\rangle=\langle\alpha|B| \alpha\rangle=0$ and therefore that $A|\alpha\rangle=B|\alpha\rangle=0$. So $|\alpha\rangle \perp \operatorname{ran}(A)$ and $|\alpha\rangle \perp \operatorname{ran}(B)$, and therefore $|\alpha\rangle \perp$ $\operatorname{ran}(A)+\operatorname{ran}(B)$. This shows that $\operatorname{ran}(A)+\operatorname{ran}(B) \subseteq \operatorname{ran}(A+B)$, and so the first claim is proven.

Now assume $[A]=[B]$. We next claim that $\left[K^{*} A K\right]=\left[K^{*} B K\right]$ for any $n \times$ $m$ matrix $K$. To see this, let $|\alpha\rangle \in \operatorname{ker}\left(K^{*} A K\right)$. Then $\left\langle\alpha\left|K^{*} A K\right| \alpha\right\rangle=0$, that is, $\left\langle A^{1 / 2} K \alpha \mid A^{1 / 2} K \alpha\right\rangle=0$, and this implies that $K|\alpha\rangle \in \operatorname{ker}\left(A^{1 / 2}\right)=\operatorname{ker}(A)$. Since $[A]=[B]$, we get $K|\alpha\rangle \in \operatorname{ker}(B)$, and therefore $|\alpha\rangle \in \operatorname{ker}\left(K^{*} B K\right)$. So we have shown that $\operatorname{ker}\left(K^{*} A K\right) \subseteq \operatorname{ker}\left(K^{*} B K\right)$. By symmetry the reverse containment also holds, so we conclude that the two kernels are equal, i.e., $\left[K^{*} A K\right]=\left[K^{*} B K\right]$.

We can now prove the lemma. We have $\Psi(C)=\sum K_{i}^{*} C K_{i}$ for some finite family of $n \times m$ matrices $K_{i}$. So, using the two claims, we have

$$
[\Psi(A)]=\left[\sum K_{i}^{*} A K_{i}\right]=\bigvee\left[K_{i}^{*} A K_{i}\right]=\bigvee\left[K_{i}^{*} B K_{i}\right]=\left[\sum K_{i}^{*} B K_{i}\right]=[\Psi(B)]
$$

as desired.

We note that a version of Lemma 7.1 for normal CP maps between von Neumann algebras can be proven using the normal Stinespring theorem ([2], Theorem III.2.2.4).

We can now describe the appropriate version of the pushforward construction for CP maps. Here we return to the "connecting mixed states" point of view; note that if $A, C \in \mathcal{M} \otimes M_{k}$ are positive then since $\Psi$ is completely positive, $\Psi(A)$ and $\Psi(C)$ will also be positive. Lemma 7.1 shows that $\mathrm{CP}$ maps preserve the relevant notion of equivalence between positive matrices.

Definition 7.2 Let $\mathcal{M} \subseteq M_{m}$ and $\mathcal{N} \subseteq M_{n}$ be unital $*$-subalgebras and let $\Phi$ : $\mathcal{M} \rightarrow \mathcal{N}$ be a CP map. Suppose $\left(\mathcal{R}_{k}\right)$ is an intrinsic quantum relation on $\mathcal{M}$. Then 
its pushforward along $\Phi$ is the intrinsic quantum relation $\left(\overrightarrow{\mathcal{R}}_{k}\right)$ on $\mathcal{N}$ defined by, for each $k \in \mathbb{N}$, letting $(P, Q)$ belong to $\overrightarrow{\mathcal{R}}_{k}$ if $\left(\left[\Phi^{*}(P)\right],\left[\Phi^{*}(Q)\right]\right)$ belongs to $\mathcal{R}_{k}$.

In order to justify this definition, we must check that $\left(\overrightarrow{\mathcal{R}}_{k}\right)$ satisfies the axioms given in Definition 5.3. This can be done directly using Lemma 7.1, but according to Theorem 5.4, it can also be done by finding a concrete quantum relation $\mathcal{W}$ on $\mathcal{N}$ with the property that $(P, Q) \in \overleftarrow{\mathcal{R}}_{k}$ if and only if $P\left(A \otimes I_{k}\right) Q \neq 0$ for some $A \in \mathcal{W}$ This will be achieved in Theorem 7.4 below. Thus, that theorem will simultaneously establish that the pushforward construction is well-defined and identify its concrete formulation.

We require two simple facts about positive matrices.

Lemma 7.3 (a) Let $A, C \in M_{m}$ be positive, let $B \in M_{n}$, and let $K_{1}, K_{2} \in M_{m, n}$. Then $K_{1}^{*} A K_{1} B K_{2}^{*} C K_{2}=0$ if and only if $A K_{1} B K_{2}^{*} C=0$.

(b) Let $A, X_{i}, Y_{j} \in M_{m}$ and suppose the $X_{i}$ and $Y_{j}$ are positive. Then $\left(\sum X_{i}\right) A\left(\sum Y_{j}\right)=$ 0 if and only if $X_{i} A Y_{j}=0$ for all $i$ and $j$.

Proof (a) The reverse implication is trivial. For the forward implication let $D=$ $B K_{2}^{*} C K_{2}$ and suppose $K_{1}^{*} A K_{1} D=0$. Then

$$
0=D^{*} K_{1}^{*} A K_{1} D=\left(A^{1 / 2} K_{1} D\right)^{*}\left(A^{1 / 2} K_{1} D\right),
$$

so $A^{1 / 2} K_{1} D=0$ and therefore $A K_{1} D=0$, i.e., $A K_{1} B K_{2}^{*} C K_{2}=0$. Applying the same argument to the adjoint of the expression $A K_{1} B K_{2}^{*} C K_{2}$ then yields the conclusion $A K_{1} B K_{2}^{*} C=0$.

(b) Again, the reverse implication is trivial. For the forward implication, we claim that if $\left(X_{1}+X_{2}\right) B=0$ with $X_{1}, X_{2} \geq 0$ then $X_{1} B=X_{2} B=0$. This inductively implies the same statement with any finite number of $X_{i}$ 's. Taking $B=A\left(\sum Y_{j}\right)$ in the statement of the lemma then yields $X_{i} A\left(\sum Y_{j}\right)=0$ for all $i$, and applying the same argument to the adjoint of each of these expressions produces the desired conclusion.

To verify the claim, suppose $\left(X_{1}+X_{2}\right) B=0$. Then

$$
0=B^{*}\left(X_{1}+X_{2}\right) B=B^{*} X_{1} B+B^{*} X_{2} B,
$$

and since both $B^{*} X_{1} B$ and $B^{*} X_{2} B$ are positive, this implies that both are zero. It follows that $X_{1} B=X_{2} B=0$, as claimed.

Theorem 7.4 Let $\mathcal{M} \subseteq M_{m}$ and $\mathcal{N} \subseteq M_{n}$ be unital $*$-subalgebras and let $\Phi$ : $\mathcal{M} \rightarrow \mathcal{N}$ be a $C P$ map given by $\Phi: B \mapsto \sum_{i=1}^{d} K_{i} B K_{i}^{*}$. Suppose $\mathcal{V} \subseteq M_{m}$ is a concrete quantum relation on $\mathcal{M}$. Then its pushforward is concretely given as the $\mathcal{N}^{\prime}-\mathcal{N}^{\prime}$ bimodule generated by

$$
\left\{K_{i} A K_{j}^{*}: A \in \mathcal{V}, 1 \leq i, j \leq d\right\}
$$

Proof Let $\mathcal{W}$ be the $\mathcal{N}^{\prime}-\mathcal{N}^{\prime}$ bimodule generated by the matrices $K_{i} A K_{j}^{*}$ for $A \in \mathcal{V}$ and $1 \leq i, j \leq d$. We must show that for any $k \in \mathbb{N}$ and any projections $P, Q \in M_{k}(\mathcal{N})$, 
we have $P\left(B \otimes I_{k}\right) Q \neq 0$ for some $B \in \mathcal{W}$ if and only if $\left[\Phi^{*}(P)\right]\left(A \otimes I_{k}\right)\left[\Phi^{*}(Q)\right] \neq 0$ for some $A \in \mathcal{V}$.

Since $P$ and $Q$ commute with anything in $\mathcal{N}^{\prime} \times I_{k}$, the condition

$$
P\left(B \otimes I_{k}\right) Q \neq 0 \text { for some } B \in \mathcal{W}
$$

obtains if and only if

$$
P\left(K_{i} A K_{j}^{*} \otimes I_{k}\right) Q \neq 0 \text { for some } A \in \mathcal{V} \text { and some } i, j .
$$

Equivalently,

$$
P\left(K_{i} \otimes I_{k}\right)\left(A \otimes I_{k}\right)\left(K_{j}^{*} \otimes I_{k}\right) Q \neq 0
$$

for some $A \in \mathcal{V}$ and some $i, j$, which by Lemma 7.3 (a) is equivalent to

$$
\left(K_{i}^{*} \otimes I_{k}\right) P\left(K_{i} \otimes I_{k}\right)\left(A \otimes I_{k}\right)\left(K_{j}^{*} \otimes I_{k}\right) Q\left(K_{j}^{*} \otimes I_{k}\right) \neq 0
$$

for some $A \in \mathcal{V}$ and some $i, j$. Then since $\Phi^{*}(P)=\sum\left(K_{i}^{*} \otimes I_{k}\right) P\left(K_{i} \otimes I_{k}\right)$ and $\Phi^{*}(Q)=\sum\left(K_{j}^{*} \otimes I_{k}\right) Q\left(K_{j} \otimes I_{k}\right)$, by Lemma 7.3 (b) the last statement is equivalent to

$$
\Phi^{*}(P)\left(A \otimes I_{k}\right) \Phi^{*}(Q) \neq 0 \text { for some } A \in \mathcal{V}
$$

which is trivially equivalent to

$$
\left[\Phi^{*}(P)\right]\left(A \otimes I_{k}\right)\left[\Phi^{*}(Q)\right] \neq 0 \text { for some } A \in \mathcal{V},
$$

as desired.

If $\mathcal{N}=M_{n}$ then its commutant is the set of scalar matrices, so that the pushforward described in Theorem 7.4 is just the linear span of the matrices $K_{i} A K_{j}^{*}$.

Once we know how to push forward quantum relations, it is easy to say what the appropriate notion of "morphism" should be: if $\mathcal{M}$ and $\mathcal{N}$ are both equipped with quantum relations $\mathcal{V}$ and $\mathcal{W}$, then a CPTP map from $\mathcal{M}$ to $\mathcal{N}$ should be considered a morphism if the pushforward $\overrightarrow{\mathcal{V}}$ of $\mathcal{V}$ is contained in $\mathcal{W}$. The classical version (which is recovered as the case where $\mathcal{M}=D_{m}$ and $\mathcal{N}=D_{n}$ ) would be a classical channel from a set $S$ of size $m$ to a set $T$ of size $n$ for which the pushforward of a given relation on $S$ is contained in a given relation on $T$.

Various definitions of quantum graph homomorphisms were proposed in [8,9,11]. Here the term "homomorphism" conflicts somewhat with classical usage, where a homomorphism between graphs is usually taken to be an actual function between the vertex sets, not a channel which could map vertices to probability distributions. An actual map between classical sets generalizes in the quantum setting to a $*$ homomorphism from $\mathcal{N}$ to $\mathcal{M}$. In the more general setting of $\mathrm{CP}$ maps we prefer the term "CP morphism": 
Definition 7.5 Let $\mathcal{M} \subseteq M_{m}$ and $\mathcal{N} \subseteq M_{n}$ be unital $*$-subalgebras equipped with intrinsic quantum relations $\left(\mathcal{R}_{k}\right)$ and $\left(\mathcal{S}_{k}\right)$, respectively. A $C P$ morphism from $\mathcal{M}$ to $\mathcal{N}$ is then a CP map $\Phi: \mathcal{M} \rightarrow \mathcal{N}$ with the property that $\overrightarrow{\mathcal{R}}_{k} \subseteq \mathcal{S}_{k}$ for all $k$.

In terms of concrete quantum relations $\mathcal{V}$ and $\mathcal{W}$ on $\mathcal{M}$ and $\mathcal{N}$, respectively, the condition would be that $\overrightarrow{\mathcal{V}} \subseteq \mathcal{W}$, where $\overrightarrow{\mathcal{V}}$ is the pushforward of $\mathcal{V}$. In particular, if $\mathcal{M}$ and $\mathcal{N}$ are matrix algebras and $\mathcal{V}$ and $\mathcal{W}$ are quantum graphs (i.e., operator systems), the concrete formulation given in Theorem 7.4 states that the condition for $\Phi$ to be a CP morphism is $K_{i} \mathcal{V} K_{j}^{*} \subseteq \mathcal{W}$ for all $i$ and $j$, which is Stahlke's condition [11]. However, the $\overrightarrow{\mathcal{R}}_{k} \subseteq \mathcal{S}_{k}$ forulation is manifestly intrinsic.

\section{Pullbacks}

There is also a way to pull quantum relations back via a CP map. Since we already know how to push quantum relations forward, one obvious solution is just to push forward using the adjoint map. This makes perfect sense in the finite-dimensional setting, but it fails in infinite dimensions when von Neumann algebras can no longer be identified with their preduals. However, there is an alternative approach which does straightforwardly generalize to infinite dimensions (with addition of the appropriate topological conditions, which are vacuous in finite dimensions). We describe this construction now.

The key question is how to use a $\mathrm{CP}$ map $\Psi=\Phi^{*}: \mathcal{N} \rightarrow \mathcal{M}$ to turn a projection in $\mathcal{M}$ into a projection in $\mathcal{N}$. We can do this using hereditary cones. A hereditary cone in $\mathcal{M}$ is a nonempty set $\mathcal{C}$ of positive matrices in $\mathcal{M}$ with the properties

(i) if $A \in \mathcal{C}$ then $a A \in \mathcal{C}$ for all $a \geq 0$

(ii) if $A, B \in \mathcal{C}$ then $A+B \in \mathcal{C}$

(iii) if $A \in \mathcal{C}$ and $0 \leq B \leq A$ then $B \in \mathcal{C}$.

If $P$ is a projection in $\mathcal{M}$ then $\mathcal{C}_{P}=\{A \in \mathcal{M}: A \geq 0$ and $P A=0\}$ is a hereditary cone, and it is not hard to check that every hereditary cone in $\mathcal{M}$ has this form. As it is easy to see that the inverse image under any $\mathrm{CP}$ map $\Psi: \mathcal{N} \rightarrow \mathcal{M}$ of a hereditary cone in $\mathcal{M}$ is a hereditary cone in $\mathcal{N}$, this shows us how to use $\Psi$ to turn a projection $P \in \mathcal{M}$ into a projection $\overleftarrow{\Psi}(P)$ in $\mathcal{N}$ : take $\overleftarrow{\Psi}(P)$ to satisfy $\mathcal{C}_{\Psi(P)}=\Psi^{-1}\left(\mathcal{C}_{P}\right)$

We can now define the pullback of a quantum relation via a $C P$ map. We continue to abuse notation by letting $\overleftarrow{\Psi}$ also denote the matrix level map which takes projections in $M_{k}(\mathcal{M})$ to projections in $M_{k}(\mathcal{N})$.

Definition 8.1 Let $\mathcal{M} \subseteq M_{m}$ and $\mathcal{N} \subseteq M_{n}$ be unital $*$-subalgebras and let $\Phi: \mathcal{M} \rightarrow$ $\mathcal{N}$ be a CP map. Suppose $\left(\mathcal{S}_{k}\right)$ is an intrinsic quantum relation on $\mathcal{N}$. Then its pullback is the intrinsic quantum relation $\left(\overleftarrow{\mathcal{S}}_{k}\right)$ on $\mathcal{M}$ defined by, for each $k \in \mathbb{N}$, letting $(P, Q)$ belong to $\overleftarrow{\mathcal{S}}_{k}$ if $(\overleftarrow{\Psi}(P), \overleftarrow{\Psi}(Q))$ belongs to $\mathcal{S}_{k}$, where $\Psi=\Phi^{*}$

As with pushforwards, we must justify this definition by showing that $\left(\overleftarrow{\mathcal{S}}_{k}\right)$ satisfies the axioms for an intrinsic quantum relation, and as in that case we will accomplish this by identifying the concrete quantum relation that corresponds to $\left(\overleftarrow{\mathcal{S}}_{k}\right)$ 
Theorem 8.2 Let $\mathcal{M} \subseteq M_{m}$ and $\mathcal{N} \subseteq M_{n}$ be unital $*$-subalgebras and let $\Phi: \mathcal{M} \rightarrow$ $\mathcal{N}$ be a $C P$ map given by $\Phi: B \mapsto \sum_{i=1}^{d} K_{i} B K_{i}^{*}$. Suppose $\mathcal{W} \subseteq M_{n}$ is a concrete quantum relation on $\mathcal{N}$. Then its pullback is concretely given as the $\mathcal{M}^{\prime}-\mathcal{M}^{\prime}$ bimodule generated by

$$
\left\{K_{i}^{*} B K_{j}: B \in \mathcal{W}, 1 \leq i, j \leq d\right\}
$$

Proof Fix $k \in \mathbb{N}$ and $P, Q \in M_{k}(\mathcal{M})$. Let $\Psi=\Phi^{*}$. We first claim that $\overleftarrow{\Psi}(P)=$ $\bigvee\left[\left(K_{i} \otimes I_{k}\right) P\right]$. To see this, recall that a positive matrix $A \in M_{k}(\mathcal{N})$ belongs to $\mathcal{C}_{\Psi}(P)$ if and only if $\Psi(A) P=0$. But $\Psi(A)=\sum\left(K_{i}^{*} \otimes I_{k}\right) A\left(K_{i} \otimes I_{k}\right)$, so by Lemma 7.3 we have $\Psi(A) P=0$ if and only if $A\left(K_{i} \otimes I_{k}\right) P=0$ for all $i$. The claim now follows from the definition of $\mathcal{C}_{\overleftarrow{\Psi}(P)}$

Now the condition for $(P, Q)$ to belong to the pullback of the intrinsic quantum relation associated to $\mathcal{W}$ is that

$$
\overleftarrow{\Psi}(P)\left(B \otimes I_{k}\right) \overleftarrow{\Psi}(Q) \neq 0
$$

for some $B \in \mathcal{W}$. By the claim and Lemma 7.3 (b), this happens if and only if

$$
\left[\left(K_{i} \otimes I_{k}\right) P\right]\left(B \otimes I_{k}\right)\left[\left(K_{j} \otimes I_{k}\right) Q\right] \neq 0
$$

for some $B \in \mathcal{W}$ and some $i, j$. This equivalent to saying that

$$
P\left(K_{i}^{*} \otimes I_{k}\right)\left(B \otimes I_{k}\right)\left(K_{j} \otimes I_{k}\right) Q \neq 0
$$

i.e.,

$$
P\left(K_{i}^{*} B K_{j} \otimes I_{k}\right) Q \neq 0,
$$

for some $B \in \mathcal{W}$. Since $P$ and $Q$ commute with $A \otimes I_{k}$ for every $A \in \mathcal{M}^{\prime}$, this last condition is equivalent to the statement that $(P, Q)$ belongs to the intrinsic quantum relation associated to the $\mathcal{M}^{\prime}$ - $\mathcal{M}^{\prime}$-bimodule generated by the matrices $K_{i}^{*} B K_{j}$. We conclude that the latter bimodule is the concrete form of the pullback of $\mathcal{W}$.

Again, in the case where $\mathcal{M}=M_{m}$, this pullback would simply be the linear span of the matrices $K_{i}^{*} B K_{j}$.

The pullback construction gives rise to an alternative version of CP morphism which is weaker than the one proposed in Definition 7.5. Namely, instead of requiring $\overrightarrow{\mathcal{R}}_{k} \subseteq$ $\mathcal{S}_{k}$ for all $k$ we could require $\mathcal{R}_{k} \subseteq \overleftarrow{\mathcal{S}}_{k}$ for all $k$. In concrete terms, the condition that $K_{i} \mathcal{V} K_{j}^{*} \subseteq \mathcal{W}$ for all $i$ and $j$ is replaced by the condition that $\mathcal{V} \subseteq \sum_{i, j} K_{i}^{*} \mathcal{W} K_{j}$. The second condition is implied by the first (multiply the first condition on the left by $K_{i}^{*}$ and on the right by $K_{j}$, then sum over $i$ and $j$ and invoke the identity $\sum K_{i}^{*} K_{i}=I_{m}$ ). Classically, the first version demands that if the point $x$ is related to the point $y$ then $x^{\prime}$ must be related to $y^{\prime}$ for any $x^{\prime}$ and $y^{\prime}$ such that the transition probabilities $x \rightarrow x^{\prime}$ 
and $y \rightarrow y^{\prime}$ are both nonzero, while the second version asks only that there be at least one such pair $\left(x^{\prime}, y^{\prime}\right)$.

Open Access This article is licensed under a Creative Commons Attribution 4.0 International License, which permits use, sharing, adaptation, distribution and reproduction in any medium or format, as long as you give appropriate credit to the original author(s) and the source, provide a link to the Creative Commons licence, and indicate if changes were made. The images or other third party material in this article are included in the article's Creative Commons licence, unless indicated otherwise in a credit line to the material. If material is not included in the article's Creative Commons licence and your intended use is not permitted by statutory regulation or exceeds the permitted use, you will need to obtain permission directly from the copyright holder. To view a copy of this licence, visit http://creativecommons.org/licenses/by/4.0/.

\section{Appendix: Superselection Rules}

A central feature of quantum mechanics is the possibility of forming superpositions of states, famously illustrated by the parable of Schrodinger's cat. However, not all superpositions are physically allowed. For instance, in elementary quantum mechanics one cannot prepare an isolated system in a superposition of two states in which different numbers of particles are present, or whose total charges are different. Such restrictions are known as "superselection rules". In their presence the Hilbert space of the system will decompose into an orthogonal sum of "superselection sectors", subspaces within which all pairs of states can be superposed.

In particular, one cannot perform a measurement on an isolated system which could result in the system being in a forbidden superposition. This means that all physical observables are restricted to have a block diagonal form such that the orthogonal projection onto any eigenspace commutes with the orthogonal projections onto the blocks. Thus, the physical observables should typically be modelled not by arbitrary self-adjoint matrices in $M_{m}$, but rather by self-adjoint matrices belonging to a fixed unital $*$-subalgebra of $M_{m}$. The significance of superselection rules in quantum information theory has been studied in $[1,4,5]$.

A qualification is in order here. When one is encoding information in a quantum mechanical system, superselection rules are only absolute if the system is isolated. Thus, in principle, one could achieve a "forbidden" superposition by first coupling the system of interest to an external system, then preparing the composite system in the desired superposition, then discarding the external system without measuring it. That is, if there is a rule forbidding superposition of the states $|\alpha\rangle$ and $\left|\alpha^{\prime}\right\rangle$ in the first system when it is isolated, one finds states $|\beta\rangle$ and $\left|\beta^{\prime}\right\rangle$ of the second system such that superposition of $|\alpha\rangle|\beta\rangle$ and $\left|\alpha^{\prime}\right\rangle\left|\beta^{\prime}\right\rangle$ is not forbidden. However, even if possible in principle, this strategy may not be feasible in practice. Indeed, the whole reason why ordinary communication is classical is exactly because one is practically unable to create quantum superpositions of macroscopic objects. Thus, operative superselection rules may arise not from fundamental physics but from limitations of the experimental apparatus.

For example, suppose that a sender populates a potential well with $n$ hydrogen atoms and transmits the system to a recipient, with the transmitted information being simply the value of $n$. If the system is isolated then states with different values of $n$ 
definitely cannot be superposed, but even if this restriction is relaxed the sender might not have the ability to create such superpositions. If so, this information is classical.

To make this example more interesting, suppose the sender has the ability to prepare the atoms in desired spin states and wants to encode information in this way. States of the system with different values of $n$ still cannot be superposed, but states with the same value of $n$ can be. In this case the relevant $*$-algebra $\mathcal{M}$ is a direct sum $\bigoplus M_{2^{n}}$ because there are $2^{n}$ possible basic spin states in a system with $n$ atoms.

Another way that $\mathcal{M}$ could be neither $M_{m}$ nor $D_{m}$ is if the transmitted information comes in two parts, a classical part and a quantum part. In that case we would have $\mathcal{M}=M_{m} \otimes D_{k}$ where $m$ is the number of degrees of freedom of the quantum part of the message and $k$ is the number of degrees of freedom of the classical part. A situation of this type could arise if, say, information is encoded in an array of heavy atoms using both their spin states and their centers of mass. (The $i$ th atom might be placed at one of two locations $(i, 1)$ or $(i, 2)$ in a $k \times 2$ grid, for example.) Models in which the spin of an atom is treated quantum mechanically while its center of mass is treated classically are familiar from standard analyses of the Stern-Gerlach experiment.

How would basic notions from quantum error correction generalize to the mixed setting? Given the abstract formulations presented earlier, this question is easy to answer. The situation would be that we have unital $*$-algebras $\mathcal{M} \subseteq M_{m}$ and $\mathcal{N} \subseteq M_{n}$ and a CPTP map $\Phi: \mathcal{M} \rightarrow \mathcal{N}$. The quantum confusability graph would then be the pullback along $\Phi$ of the diagonal quantum relation on $\mathcal{N}$, i.e., its commutant $\mathcal{N}^{\prime}$. If $\Phi$ has the form $\Phi: B \mapsto \sum_{i=1}^{d} K_{i} B K_{i}^{*}$, then according to Theorem 8.2 this pullback would be the $\mathcal{M}^{\prime}-\mathcal{M}^{\prime}$ bimodule generated by $\left\{K_{i}^{*} B K_{j}: B \in \mathcal{N}^{\prime}\right\}$.

As a simple special case, suppose $\mathcal{M}=M_{m} \otimes D_{m^{\prime}} \subseteq M_{m m^{\prime}}$ and $\mathcal{N}=M_{n} \otimes D_{n^{\prime}} \subseteq$ $M_{n n^{\prime}}$. Then $\mathcal{M}^{\prime}=I_{m} \otimes D_{m^{\prime}}$ and $\mathcal{N}^{\prime}=I_{n} \otimes D_{n^{\prime}}$, and the quantum confusability graph is the $\mathcal{M}^{\prime}-\mathcal{M}^{\prime}$ bimodule generated by $\left\{K_{i}^{*}\left(I_{n} \otimes B\right) K_{j}: B \in D_{n^{\prime}}\right\}$. This expression can be made more explicit if the Kraus matrices are chosen in a natural way. Namely, for each $1 \leq a \leq m$ and $1 \leq b \leq n$ the map $\Phi$ induces a CP map from $M_{m} \cong M_{m} \otimes E_{a a} \subseteq M_{m m^{\prime}}$ to $M_{n} \cong M_{n} \otimes E_{b b} \subseteq M_{n n^{\prime}}$, where $E_{a a}$ is the $m^{\prime} \times m^{\prime}$ matrix with a 1 in the $(a, a)$ entry and 0's elsewhere and $E_{b b}$ is the $n^{\prime} \times n^{\prime}$ matrix with a 1 in the $(b, b)$ entry and 0 's elsewhere. Thus, for each $a$ and $b$ we can find a family of $n \times m$ Kraus matrices $K_{i}^{a b}$ such that $\Phi(B)=\sum_{a, b, i}\left(K_{i}^{a b} \otimes E_{a a}\right) B\left(K_{i}^{a b} \otimes E_{a a}\right)^{*} \otimes E_{b b}$, and the condition that $\Phi$ be trace preserving is $\sum_{a, b, i}\left(K_{i}^{a b}\right)^{*} K_{i}^{a b} \otimes E_{a a}=I_{m m^{\prime}}$, or equivalently, $\sum_{b, i}\left(K_{i}^{a b}\right)^{*} K_{i}^{a b}=I_{m}$ for each $a$ (a version of stochasticity). With these Kraus matrices, the quantum confusability graph is the operator system $\mathcal{V}$ spanned by the matrices $\left(K_{i}^{a b}\right)^{*} K_{j}^{a^{\prime} b} \otimes E_{a a^{\prime}} \in M_{m m^{\prime}}$ for arbitrary $a, a^{\prime}, b, i$, and $j$, which is automatically an $\mathcal{M}^{\prime}-\mathcal{M}^{\prime}$ bimodule.

In this setting a quantum code would be a projection $E \in \mathcal{M}$ such that $E \mathcal{V} E=I_{m} \otimes$ $D_{m^{\prime}}$, where $\mathcal{V}$ is the quantum confusability graph just described. Concretely, we can write $E=\sum P_{a} \otimes E_{a a}$ where each $P_{a}$ is a projection in $M_{m}$, and the error correction conditions would state that (1) for each $a, b, i$, and $j$ the matrix $P_{a}\left(K_{i}^{a b}\right)^{*} K_{j}^{a b} P_{a}$ is a scalar multiple of $P_{a}$, and (2) for each $a \neq a^{\prime}, b, i$, and $j$ the matrix $P_{a}\left(K_{i}^{a b}\right)^{*} K_{j}^{a^{\prime} b} P_{a^{\prime}}$ is zero. 


\section{References}

1. Bartlett, S.D., Wiseman, H.M.: Entanglement constrained by superselection rules. Phys. Rev. Lett. 91, 4 (2003)

2. Blackadar, B.: Operator Algebras: Theory of $\mathrm{C}^{*}$-Algebras and Von Neumann Algebras. Springer, Berlin (2006)

3. Duan, R., Severini, S., Winter, A.: Zero-error communication via quantum channels, noncommutative graphs, and a quantum Lovász number. IEEE Trans. Inf. Theory 59, 1164-1174 (2013)

4. Jones, S.J., Wiseman, H.M., Bartlett, S.D., Vaccaro, J.A., Pope, D.T.: Entanglement and symmetry: a case study in superselection rules, reference frames, and beyond. Phys. Rev. A 74, 16 (2006)

5. Kitaev, A., Mayers, D., Preskill, J.: Superselection rules and quantum protocols. Phys. Rev. A 69, 20 (2004)

6. Knill, E., Laflamme, R.: Theory of quantum error-correcting codes. Phys. Rev. A 55, 900-911 (1997)

7. Kuperberg, G., Weaver, N.: A von Neumann algebra approach to quantum metrics. Mem. Am. Math. Soc. 215(v), 1-80 (2012)

8. Ortiz, C. M., Paulsen, V. I.: Quantum graph homomorphisms via operator systems. arXiv:1505.00483

9. Roberson, D. E., Mancinska, L.: Graph homomorphisms for quantum players. arXiv:1212.1724

10. Shirokov, M.E., Shulman, T.V.: On superactivation of one-shot quantum zero-error capacity and the related property of quantum measurements. Probl. Inf. Transm. 50, 232-246 (2014)

11. Stahlke, D.: Quantum zero-error source-channel coding and non-commutative graph theory. IEEE Trans. Inf. Theory 62, 554-577 (2016)

12. Weaver, N.: Quantum relations. Mem. Am. Math. Soc. 215(v-vi), 81-140 (2012)

Publisher's Note Springer Nature remains neutral with regard to jurisdictional claims in published maps and institutional affiliations. 